\title{
Quake-spotting 'telescope' may map North American continent
}

[SEATTLE] A consortium of 90 US universities is planning an unprecedented new seismology facility to map North America. It will use a network of portable devices that its supporters describe as a form of Hubble telescope looking deep into the Earth.

Funding for the experimental facility, currently called USArray, is being sought by Incorporated Research Institutions for Seismology (IRIS). The consortium oversees major capital expenditure for member universities' research projects from agencies such as the National Science Foundation (NSF).

"The heart of the facility is a transportable, dense array of seismometers, which will improve the quality of seismic images of the continental lithosphere and deeper mantle by an order of magnitude," says a report from a workshop held by IRIS in March in Albuquerque, New Mexico.

The report says that a series of deployments of such an array would cover the contiguous United States in ten years, and result in a uniform data set that would be ideal for imaging the continent at high spatial resolution. The array will use new communication technologies, it adds, allowing data to be made available in real-time on the Internet.

The NSF has already included the anticipated costs - estimated to be tens of millions of dollars - in its long-range budget plans. IRIS intends to send a report on USArray to the foundation in the next month. NSF officials say they expect to brief the advisory National Science Board on the project in the autumn, and seek funding from Congress during hearings for the 2001 budget.

To muster support, IRIS scientists briefed representatives from the Council of the National Seismic System, a group of some 30 seismology networks that held its annual meeting in Seattle last week.

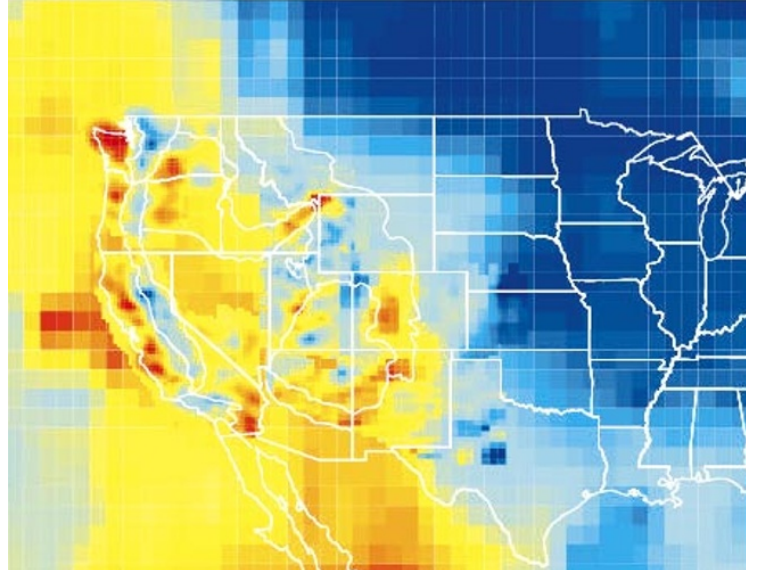

Looking into the depths: this composite image of upper mantle P-wave seismic structure, some $100 \mathrm{~km}$ below the western United States, shows more variation than seismologists had previously suspected. Red areas show the partially molten, low-velocity mantle; blue represents subsolidus, high-velocity mantle.

Seismologists say the array could provide researchers with a rich catalogue of information about the structure and formation of the continent, while offering insights into such topics as how surface water flows, the safety of sites for waste disposal, and more explanations of mineral and oil deposits.

"It is an opportunity to really understand the continent we live on," says IRIS's president David Simpson. He calls the experiment "a major challenge" - a reference to the logistics of collecting and managing the data.

If USArray is funded and set up, proponents hope to coordinate their efforts with seismologists in Canada and Mexico to expand mapping of the Earth's crust.

USArray would be the biggest project undertaken by IRIS (which has an annual operating budget of about $\$ 11$ million) since it was created 16 years ago to combine member institutions' resources to advance seismology research cost-efficiently.

Seismologists touted the facility's opportunities enthusiastically at the Seattle meeting. But they are realistic about the federal government's funding process. USArray

\section{Search for a key to secrets of the Universe}

[LONDON] Scientists at the Stanford Linear Accelerator Center in California started a search on Tuesday (11 May) for evidence of short-lived subatomic particles known as B-mesons, from collisions of electrons and positrons.

The B-mesons and their anti-particles will be detected using a 1,200-tonne particle detector known as BaBar, positioned where the electron and positron beams meet.
The experiment is designed to find clues as to why the Universe has more matter than antimatter.

Scientists believe that microseconds after the Big Bang, equal amounts of matter and anti-matter should have annihilated each other. "Mysteriously, a bit of matter was left over," says Ken Peach of the UK Rutherford Appleton Laboratory, one of the centres involved in the experiment. "By studying particles and their antiparticles we hope to see the tiny differences in behaviour between matter and antimatter, which will help explain the huge imbalance in the Universe," adds Peach.

BaBar, which cost $\$ 300$ million, is the result of collaborative work by more than 500 physicists and engineers from 10 countries.

Ehsan Masood faces "a long road ahead" before it is funded, they noted, and a delay in creating the facility is a distinct possibility. It also faces obstacles from within and without seismology ranks.

Steve Malone, for example, a University of Washington seismologist who chaired the Council of the National Seismic System for the past year, told the assembly of more than 50 scientists that it would require seismologists to think more collectively than in the past. Malone warned of the importance of avoiding turf wars, data-hogging and seismologists' tendency to consider an earthquake that they identify as "my earthquake".

Anne S. Meltzer, a seismologist at Lehigh University, Pennsylvania, who chairs IRIS's executive committee, added that USArray could "expand the culture of shared and coordinated resources within Earth science".

The USArray will require an unprecedented number of seismometers from the few companies that make the very broadband, high dynamic range seismometers that are required. At least 400 seismometers will be required for the portable array — passive equipment that sits on temporary pads, recording waves from near and distant earthquakes to image the Earth's crust and mantle.

According to a report on a USArray workshop held in March by Peter Shearer, of the Scripps Institution of Oceanography in San Diego, and colleagues, the facility "will provide an opportunity for local universities and research groups to target specific geologic problems". A regional pre-deployment workshop would be held for each stage, to help plan experiments and coordinate educational outreach efforts.

"Whenever a new source of seismic data becomes available, unexpected discoveries have often overshadowed the original purpose of the data collection. Almost certainly USArray will yield many such bonuses," say Shearer and his colleagues. 